\title{
Notch1 pathway in adrenocortical carcinomas: correlations with clinical outcome
}

\author{
Cristina L Ronchi, Silviu Sbiera', Barbara Altieri, Sonja Steinhauer, Vanessa Wild ${ }^{2,3}$, \\ Michaela Bekteshi, Matthias Kroiss ${ }^{3}$, Martin Fassnacht and Bruno Allolio \\ Endocrine and Diabetes Unit, Department of Internal Medicine I, University Hospital, University of Wuerzburg, \\ Oberrduerrbacher-Strasse 6, 97080 Wuerzburg, Germany \\ ${ }^{1}$ Central Laboratory, University Hospital of Wuerzburg, Wuerzburg, Germany \\ ${ }^{2}$ Institute of Pathology, University of Wuerzburg, Wuerzburg, Germany \\ ${ }^{3}$ Comprehensive Cancer Center Mainfranken, Wuerzburg, Germany
}

\author{
Correspondence \\ should be addressed \\ to C L Ronchi \\ Email \\ Ronchi_c@ukw.de
}

\begin{abstract}
Previous SNP array analyses have revealed genomic alterations of the Notch pathway as being the most frequent abnormality in adrenocortical tumors (ACTs). The aim of the present study was to evaluate the expression of components of Notch signaling in ACTs and to correlate them with clinical outcome. The mRNA expression of JAG1, NOTCH1, and selected target genes of NOTCH1 (HES1, HES5, and HEY2) was evaluated in 80 fresh frozen samples (28 normal adrenal glands (NAGs), 24 adenomas (ACAs), and 28 carcinomas (ACCs)) by quantitative RT-PCR. Immunohistochemistry was performed in 221 tissues on paraffin slides (16 NAGs, 27 ACAs, and 178 ACCs) for JAG1, activated NOTCH1 (aNOTCH1), and HEY2. An independent ACC validation cohort $(n=77)$ was then also investigated. HEY2 mRNA expression was higher in ACCs than it was in ACAs $(P<0.05)$. The protein expression of all of the factors was high (H-score 2-3) in a larger proportion of ACCs as compared to ACAs and NAGs (JAG1 in 27, 15, and 10\%; aNOTCH1 in 13, 8, and 0\%; HEY2 in 66, 61, and 33\% respectively, all $P<0.001)$. High JAG1 expression was associated with earlier tumor stages and lower numbers of metastases in ACCs (both $P=0.08$ ) and favorably impacted overall and progression-free survival (PFS) (131 vs 30 months, hazard ratio (HR) 0.45, and 37 vs 9 months, HR 0.51, both $P<0.005)$. This impact on overall survival (OS) was confirmed in the validation cohort. No such association was observed for aNOTCH1 or HEY2. In conclusion, different components of the Notch1 signaling pathway are overexpressed in ACCs, which suggests a role for the pathway in malignant transformation. However, JAG1 is overexpressed in a subgroup of ACCs with a better clinical outcome.
\end{abstract}

\section{Key Words}

- adrenocortical tumors

- Notch1 pathway

- JAG1

-Wnt/ $\beta$-catenin

\section{Introduction}

The pathogenesis of both benign adrenocortical adenomas (ACAs) and carcinomas (ACCs) remains incompletely understood (Fassnacht et al. 2013, Else et al. 2014) despite major recent advances (Assie et al. 2014). In a previous study that employed SNP array profiling in adrenocortical tumors (ACTs), we identified the Notch1 signaling pathway as being the most frequently altered pathway in both ACAs and ACCs, followed by alterations 
in Wnt/ $\beta$-catenin signaling (Ronchi et al. 2013). This observation suggests a major role of Notch signaling in adrenocortical tumorigenesis.

The Notch signaling pathway regulates cell-fate decisions throughout embryonic development and adult life by controlling neurogenesis, angiogenesis, apoptosis, cell cycle, proliferation, and differentiation (Capaccione \& Pine 2013). Notch is a transmembrane receptor with an extracellular domain that possesses epidermal growth factor repeats and an intracellular domain that contains a nuclear localization sequence (NICD), an RBP-Jк-associated module (RAM) domain, a C-terminal PEST region, and seven ankyrin repeats. Up until now, four receptors (NOTCH1, NOTCH2, NOTCH3, and NOTCH4) and six ligands (JAG1, JAG2, DLL1, DLL3, DLL4, and DLK1) have been identified. After the binding of a ligand to the respective receptor, $\gamma$-secretase complex mediates the cleavage of the transmembrane domain of the Notch receptor to release the NICD, which then translocates into the nucleus and activates the transcription of several target genes, including the hairy enhancer of split (HES) family, the HES-related (HEY) family, and many others involved in apoptosis (NFK2, CDKN1A, BIRC5/surviving, BCL2), cell cycle or proliferation (CCND1, DTX1, p21/WAF1, CDKN1A$B, I G F R)$, transcription (CMYC, GATA3), or with unknown function (NOTCH3, PTCRA) (Supplementary Figure S1, see section on supplementary data given at the end of this article) (Ranganathan et al. 2011, Chillakuri et al. 2012).

Dysregulation of the Notch signaling pathway has been implicated in several human cancers. For instance, the translocation $\mathrm{t}(7 ; 9)(\mathrm{q} 34 ; \mathrm{q} 34.3)$ or mutations in the NOTCH gene (including PEST truncating mutations), which lead to Notch-ligand independent activation or to impaired degradation of activated Notch, have been shown to play a role in T-cell acute lymphoblastic leukemia (Weng et al. 2004, Jundt et al. 2008, Ferrando 2010, Paganin \& Ferrando 2011). In solid tumors, both oncogenic actions or a tumor suppressor role of the Notch signaling pathway and its components have been reported, depending on the cell type and context (Supplementary Table S1, see section on supplementary data given at the end of this article) (Radtke \& Raj 2003, Balint et al. 2005, Wang et al. 2006, Westhoff et al. 2009, Lobry et al. 2011, Mazur et al. 2012, Rizzo et al. 2013, Carvalho et al. 2014, Du et al. 2014). In most cases, activated Notch signaling is associated with more aggressive behavior and poorer prognosis (Capaccione \& Pine 2013). However, in some malignancies, NOTCH1 activation may also induce cell growth arrest (Wang et al. 2007).
Recent data have suggested that Notch works as a hub that enables cross-talk among different oncogenic pathways, such as the Wnt/ $\beta$-catenin signaling, the Sonic hedgehog (Shh), and the AKT/PI3K pathways (Supplementary Figure S1, see section on supplementary data given at the end of this article). In particular, the link between Notch and Wnt/ $\beta$-catenin signaling has been investigated in human development (Balint et al. 2005, Crosnier et al. 2006, Yamamizu et al. 2010, Peignon et al. 2011, Ravindran \& Devaraj 2012, Gopalakrishnan et al. 2014), and either additive or opposing effects have been reported, depending on the respective tissue and interfering factors (Kwon et al. 2011, Kim et al. 2012). Interestingly, Notch pathway activation has also been related, either alone or together, to $\mathrm{Wnt} / \beta$-catenin activation and p53 deletion, to the epithelial-mesenchymal transition that is involved in the initiation of metastasis (Wang et al. 2009a, Espinoza \& Miele 2013, Chanrion et al. 2014), and to resistance to treatment (Wang et al. 2009b, Yao \& Qian 2010, Ma et al. 2013, Yoon et al. 2014).

Because of its oncogenic role in many cancers, inhibitors of the Notch pathway have been developed either to act at the level of $\gamma$-secretase or to bind to Notch ligands or receptors, thereby inhibiting Notch activation and suppressing tumor cell growth (Supplementary Figure S1, see section on supplementary data given at the end of this article) (Groth \& Fortini 2012, Capaccione \& Pine 2013, Espinoza \& Miele 2013, Gordon \& Aster 2014, Previs et al. 2014). Several clinical trials with these compounds alone or in combination are currently ongoing or have been recently completed (ClinicalTrials. gov) (Richter et al. 2014, Lee et al. 2015, LoConte et al. 2015, Messersmith et al. 2015). Moreover, inhibiting Notch signaling (i.e., by pretreatment) has been shown to sensitize tumors to platinum compounds or other cytotoxic drugs, such as gemcitabine (Meng et al. 2009, Wang et al. 2010, McAuliffe et al. 2012). Finally, because the Notch ligand JAG1 is overexpressed in many cancers and plays an important role in tumor biology (Steg et al. 2011), targeting JAG1 directly may represent a promising therapeutic tool (Dai et al. 2014, Li et al. 2014).

Adrenal gland morphology and functioning are deeply interconnected, and signaling pathways, such as the Wnt/ $\beta$-catenin, Shh, and Notch pathways, are required to preserve the integrity of their functioning (Gallo-Payet \& Battista 2014). In particular, constitutive activation of the Wnt/ $\beta$-catenin signaling pathway has been demonstrated to be an early step in adrenocortical tumorigenesis (Tissier et al. 2005, El Wakil \& Lalli 2011, Gaujoux et al. 2011), but so far only limited data are available on the

Published by Bioscientifica Ltd 
involvement of Notch signaling. However, recurrent copy number gains in the $J A G 1$ (frequency $>50 \%$ ) or $J A G 2$ gene (frequency $>40 \%$ ) have been found in ACCs, which suggests a role for these genes in malignant transformation (Ronchi et al. 2013). Furthermore, Simon et al. (2012) demonstrated that the JAG1 gene is overexpressed in ACCs (as compared to normal adrenal glands (NAGs) and ACAs) and that JAG1 up-regulation in Y1 mouse cells is able to enhance cell proliferation and aggressiveness through the activation of Notch signaling in adjacent cells.

The major aim of the present study was therefore to more comprehensively investigate the components of the Notch pathway and their relation to Wnt/ $\beta$-catenin signaling in NAGs and in a large series of ACTs in order to characterize their potential role in adrenocortical tumorigenesis. Furthermore, we also investigated the relationship between the expression of Notch-related factors and clinical outcome in patients with ACC.

\section{Materials and methods}

\section{Tissue samples, patients, and clinical annotations}

Eighty fresh frozen adrenal tissues (28 NAGs, 24 ACAs, and 28 ACCs) were used for the evaluation of mRNA levels of several components of the Notch and Wnt/ $\beta$-catenin pathways.

A series of 236 tissue samples on paraffin slides comprising 16 NAGs (including seven adrenal hyperplasia), 27 ACAs, 178 ACCs, and 15 other tissues serving as controls (including pancreas, colon, prostate, and ovarian cancer) was evaluated by immunohistochemistry. Among the ACC samples, 135 were obtained from surgery of the primary tumor, 26 from local recurrences, and 17 from distant metastases. In that series, 16 of the ACCs had been also investigated as part of a previous SNP array analysis and were used for the comparison between $\mathrm{CN}$ alterations and protein expression (Ronchi et al. 2013).

Clinical parameters, such as sex, age at diagnosis, date of surgery, tumor size, results of hormone analysis, and, in the case of ACC, tumor stage according to the European Network for the Study of Adrenal Tumors (ENSAT) classification (Fassnacht et al. 2009), Weiss score, Ki67 proliferation index, presence and number of distant metastases, and detailed follow-up information were collected through the German ACC and ENSAT registries (www.ens@t.org/registry). Malignancy and hormonal hypersecretion were defined according to established clinical, biochemical, and morphological criteria (Nieman et al. 2008). The baseline patient and tumor characteristics of both series are given in Table 1. Concerning treatment, 26 ACC patients underwent follow-up only, 34 received mitotane monotherapy (adjuvant or palliative), and 62 underwent chemotherapy with different drug combinations, including platinum compound-based protocols, streptozotocin, gemcitabine plus capecitabine, and others. Thirteen ACC patients were lost to follow-up (unknown treatment).

An independent validation cohort of 77 ACCs (63 obtained from surgery of the primary tumor, six from local recurrences, and eight from distant metastases) was also investigated in a second step by immunohistochemistry to confirm the key results of the first series.

The present study was approved by the ethics committee of the University of Wuerzburg (nos 93/02 and 88/11), and written informed consent was obtained from all of the patients.

\section{NOTCH1 mutation analysis}

A total of 46 fresh frozen ACTs (21 ACAs and 25 ACCs) were investigated for the presence of the somatic mutation in exon 34.1 of the NOTCH1 gene, which disrupts the PEST region (c.7544_7545delCT) and leads to constitutive activation. Total tumor DNA was extracted, and mutation analysis was performed by direct Sanger sequencing analysis of PCR products obtained using primer sequences and PCR conditions as previously published (Fabbri et al. 2011, Rossi et al. 2012).

\section{Gene expression analysis}

mRNA expression in fresh frozen tissue was investigated by real-time quantitative RT-PCR (qRT-PCR). Among the Notch-related factors, we choose the Notch ligand JAG1 because of previous observations in ACTs as well as the most well-known Notch-specific target genes of the HES/HEY family (HES1, HES5, and HEY2) (Ranganathan et al. 2011, Chillakuri et al. 2012). For the Wnt/ $\beta$-catenin/TCF/LEF1 axis, we selected the well-characterized target gene LEF1. In brief, RNA was isolated from fresh frozen tissue samples with the RNeasy Lipid Tissue Minikit (Qiagen) and RT with the QuantiTect Reverse Transcription Kit (Qiagen). Predesigned Taqman (Applied Biosystems, Darmstadt, Germany) gene expression assays for JAG1 (Hs01070032_m1), NOTCH1 (Hs01062014_m1), CTNNB1 (Hs00355049_m1), HES1 (Hs00172878_m1), HES5 (Hs01387463_g1), HEY2 (Hs00232622_m1), and LEF1 (Hs01547250_m1) were purchased from Applied Biosystems. Endogenously expressed $\beta$-actin (Hs9999903_m1)

Published by Bioscientifica Ltd 
Table 1 Clinical and histopathological characteristics

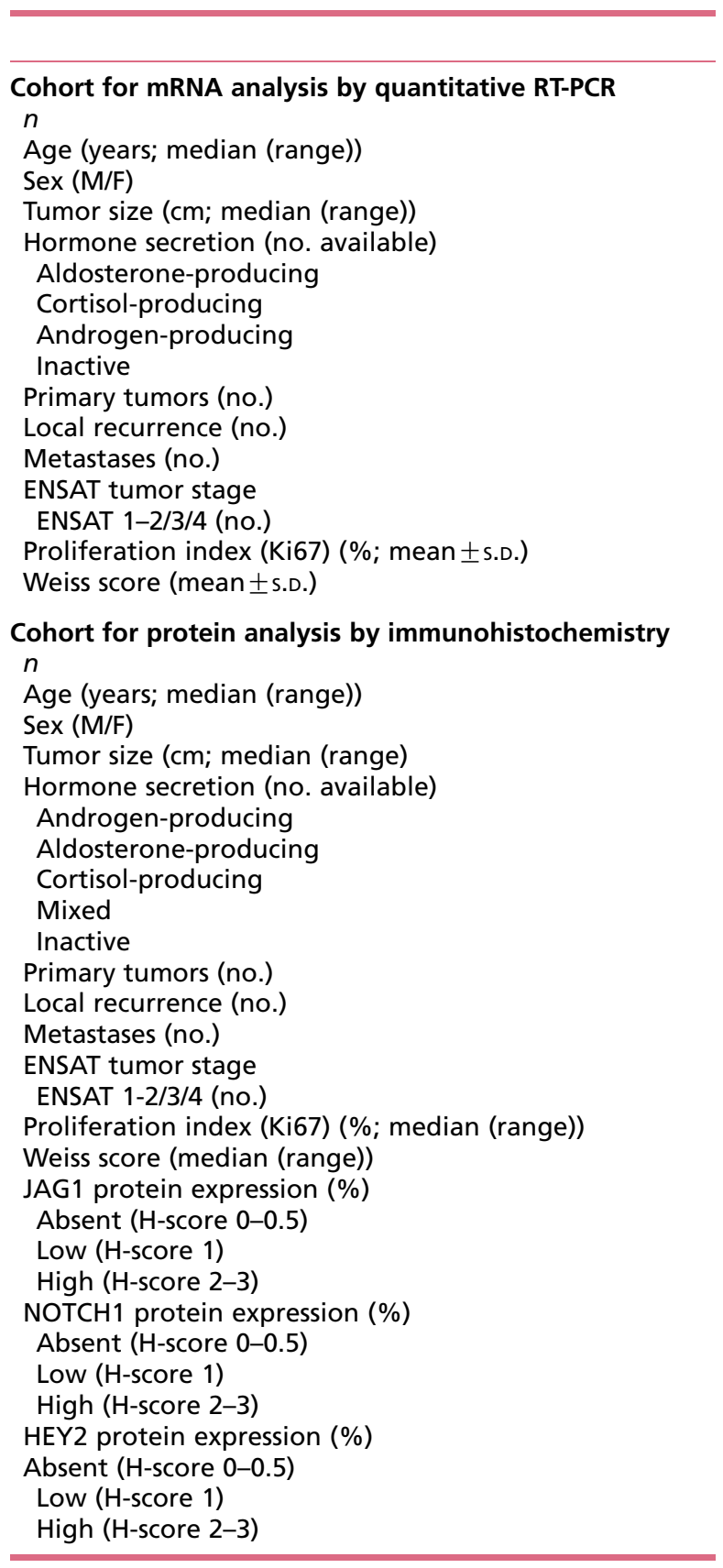

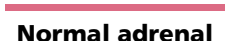

Normal adrenal

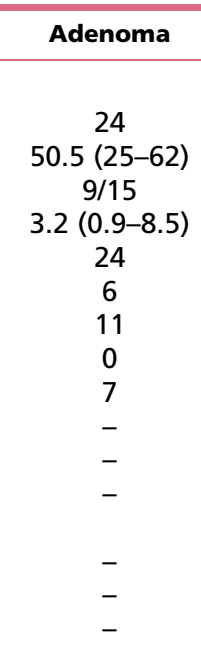

16

64 (27-79)

$-$

$-$

$-$

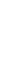

$-$

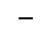

$-$

$-$

$-$

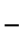

$-$

-

-

40

50

10

80

20

0

27

40

33

\begin{tabular}{ccc}
\hline Carcinoma & & $\boldsymbol{P}$ \\
28 & & \\
$20(24-78)$ & & NS \\
$14 / 14$ & & NS \\
$8(2.5-24)$ & & $<0.05$ \\
28 & & NS \\
1 & NS \\
12 & NS \\
3 & NS \\
12 & & NS \\
19 & & - \\
4 & & - \\
3 & & - \\
$13 / 10 / 4$ & & - \\
$17 \pm 10.5$ & & - \\
$6 \pm 2$ & &
\end{tabular}

NS, not significant.

was used for normalization. Forty nanograms cDNA was used for each PCR reaction, and each sample was performed in duplicate. Transcript levels were determined with the TaqMan Gene Expression Master Mix (Applied Biosystems), the CFX96 real-time thermocycler (Bio-Rad), and Bio-Rad CFX Manager 2.0 Software. Cycling conditions were $3 \mathrm{~min}$ at $95^{\circ} \mathrm{C}$ followed by 50 cycles of $30 \mathrm{~s} \mathrm{at}$ $95^{\circ} \mathrm{C}, 30 \mathrm{~s}$ at $60^{\circ} \mathrm{C}$, and $30 \mathrm{~s}$ at $72^{\circ} \mathrm{C}$. Using the $\Delta \mathrm{CT}$ method (Pfaffl 2001), the gene expression levels were normalized to those of $\beta$-actin, as previously described (Ronchi et al. 2012).

\section{Immunohistochemistry}

According to previous observations and to mRNA expression results, we selected for immunohistochemistry the Notch ligand JAG1, the activated NOTCH1 (aNOTCH1), and the specific Notch target gene HEY2. 
A total of 263 paraffin-embedded specimens, including 76 standard full slides and 187 samples assembled into three tissue microarrays (TMAs) were investigated by immunohistochemistry. TMA samples were included in the analysis only if two or more evaluable cores per patient were available after the staining procedure. Thus, the final series included 236 tissue samples (16 NAGs, 27 ACAs, 178 ACCs, and 15 positive controls; see 'Tissue samples' above). The validation cohort consisted of 77 ACCs distributed on three new TMAs.

\section{Immunostaining for JAG1, activated NOTCH1, and} HEY2 TMA and full sections were deparaffinized, and immunohistochemical detection was performed with an indirect immunoperoxidase technique after high temperature antigen retrieval in $10 \mathrm{mM}$ citric acid monohydrate buffer ( $\mathrm{pH}$ 6.5) in a pressure cooker for 13 min. Blocking of unspecific protein-antibody interactions was performed with 20\% human $A B$ serum in PBS for $1 \mathrm{~h}$ at room temperature. Primary antibody for JAG1 was a monoclonal anti-rabbit antibody (EPR4290, Lifespan Bioscience, Seattle, WA, USA) at a dilution of $1: 300$ at $4{ }^{\circ} \mathrm{C}$. Primary antibody for aNOTCH1 was a polyclonal anti-rabbit antibody against the cleaved NICD (ab 8925, epitope: VLLSRKRRRQHGQC, Abcam, Cambridge, UK) at a dilution of $1: 200$ at $4{ }^{\circ} \mathrm{C}$. Primary antibody for HEY2 was a polyclonal anti-rabbit antibody (HPA030205, SigmaAldrich) at a dilution of $1: 100$ at $4{ }^{\circ} \mathrm{C}$, together with N-Universal Negative Control Anti-Rabbit (Dako, Glostrup, Denmark). Signal amplification was achieved by En-Vision System Labeled Polymer-HRP Anti-Rabbit (Dako) for $40 \mathrm{~min}$ and was then developed for $10 \mathrm{~min}$ with DAB Substrate Kit (Vector Laboratories, Burlingame, CA, USA) according to the manufacturer's instructions. Nuclei were counterstained with Mayer's hematoxilin for 2 min. For positive controls, sections with colon cancer, pancreatic cancer, and prostate cancer were chosen, whereas cells of the tumor stroma served as internal negative controls.

All of the slides were analyzed independently by two investigators blinded to clinical information (CLR and SSt).
Both nuclear and cytoplasmic staining was evaluated according to the expected localization (cytoplasmatic and membranous for JAG1, nuclear for aNOTCH1 and HEY2), and staining intensity was graded as negative (0), low (1), medium (2), or strong (3). The percentage of positive tumor cells was calculated for each specimen and scored as 0 if $0 \%$ were positive, as 0.1 if $1-9 \%$ were positive, as 0.5 if $10-49 \%$ were positive, and as 1 if $50 \%$ or more were positive. A semiquantitative $\mathrm{H}$-score was then calculated by multiplying the staining intensity grading score with the proportion score as described previously (Ronchi et al. 2009). When discrepancies were observed, results were jointly assessed by both investigators and the final score was formed by consensus. Inter-observer agreement was strong, with a Pearson's correlation coefficient of 0.83 (95\% CI 0.78-0.87) for JAG1 and 0.67 (95\% CI 0.58-0.75) for aNOTCH1.

Immunostaining for $\boldsymbol{\beta}$-catenin Immunohistochemistry for $\beta$-catenin had been previously performed on the present TMAs, and the results have already been published elsewhere (Gaujoux et al. 2011, Ronchi et al. 2012). In brief, primary antibody was provided by BD Bioscience (1:400; San Jose, CA, USA), and the nuclear staining (representative of $\beta$-catenin pathway activation) was assessed as previously described (Gaujoux et al. 2011). A total of 59 cases among those assembled in the TMAs with fewer than two evaluable cores were excluded from the final series (seven ACAs, 50 ACCs, and two NAs), which left a final series of 144 samples.

\section{Statistical analysis}

Fisher's exact test or $\chi^{2}$ test was used to investigate dichotomic variables, whereas two-sided t-test (or nonparametric test) was used to test continuous variables. Non-parametric Kruskal-Wallis test, followed by Bonferroni post hoc test, was used for comparison among several groups for non-normal distributed variables. Correlations and 95\% CIs between different parameters were evaluated by linear regression analysis. OS was defined as the time
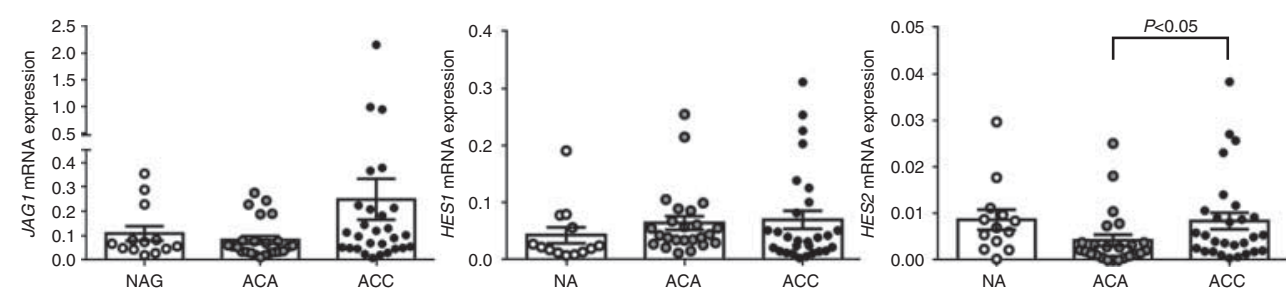

Figure 1

Relative mRNA expression of JAG1, HES1, and HEY2 in normal adrenal glands (NAGs, $n=28)$, adenomas (ACAs, $n=24)$, and carcinomas (ACCs, $n=28$ ). 
from the date of primary surgery to specific death or last follow-up, whereas PFS was defined as the time from the date of primary tumor resection to the first radiological evidence of any kind of disease progression or relapse or death. Time to progression (TTP) during therapy was defined as the time from the date of first administration to the first radiological evidence of any kind of disease progression or relapse or death. All survival curves were obtained by Kaplan-Meier estimates, and the differences between survival curves were assessed by the log-rank (Mantel-Cox) test. For the calculation of hazard ratios (HRs), two ACC groups with low or high protein expression were considered (JAG1 high-expression H-score $\geq 1$; HEY2 H-score $\geq 2$ ). A multivariate regression analysis was performed by Cox proportional hazard regression model to identify those factors that might independently influence survival. Statistical analyses were made using
GraphPad Prism version 5.0 (La Jolla, CA, USA) and SPSS Software PASW Version 21.0 (SPSS, Inc., Chicago, IL, USA). $P$ values of $<0.05$ were considered statistically significant.

\section{Results}

\section{NOTCH1 mutation analysis}

None of the 46 investigated ACTs (21 ACAs, 25 ACCs) had the known activating somatic mutation of the PEST region of the NOTCH1 gene (c.7544_7545delCT).

\section{mRNA expression of JAG1 and other Notch-related factors}

Considering all of the 80 samples together (NAGs, ACAs, and ACCs), the mRNA expression of HES1 and HEY2 was positively correlated with NOTCH1 $(R=0.46, P<0.005$,
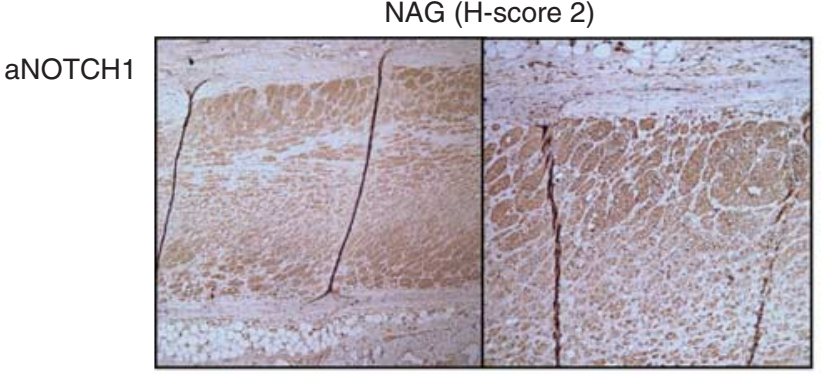

B JAG1

Capsule zona glomerulosa
(mineralcorticoids)

Zona fasciculata (glucocorticoids)

Zona reticularis (androgens)

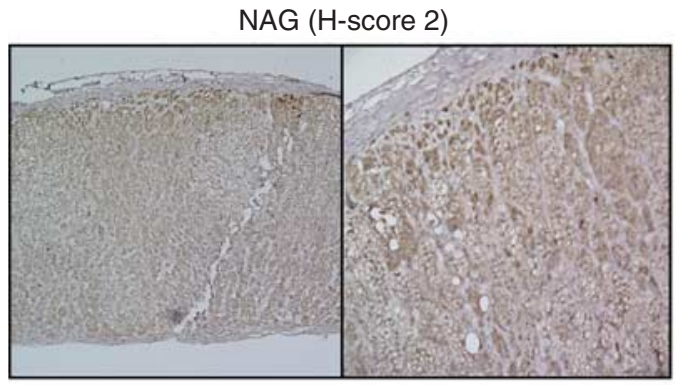

NAG (H-score 2)

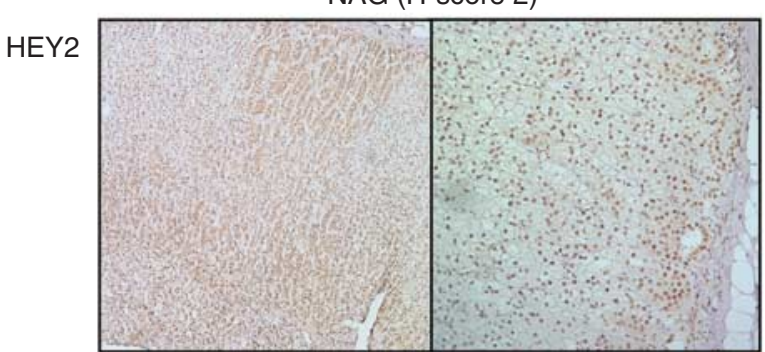

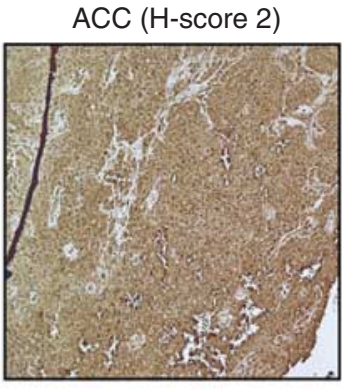

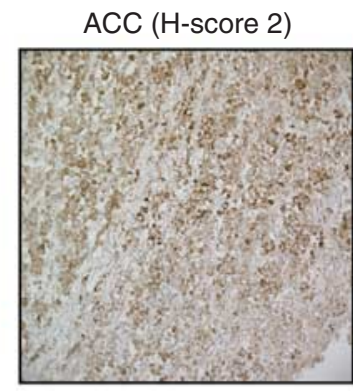

ACC (H-score 2)

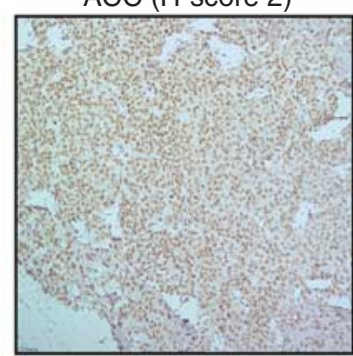

\section{Figure 2}

Examples of immunostaining of activated NOTCH1 (aNOTCH1) (A), JAG1 (B), and HEY2 (C) in a normal adrenal gland (NAG) and an adrenocortical carcinoma (ACC) (H-score 2). 
and $R=0.22, P=0.077$, respectively), JAG1 was positively correlated with both HES1 and HEY2 $(R=0.28, P<0.05$, and $R=0.24, P=0.05$, respectively), and LEF1 was positively correlated with CTNNB1 $(R=0.72, P<0.005)$, which indicates that the up-regulation of upstream signaling results in enhanced target gene expression.

HEY2 mRNA expression was significantly higher in ACCs than in ACAs $(0.0084 \pm 0.0094$ vs $0.0042 \pm 0.0060$, $P<0.05$ ), whereas JAG1 and HES1 showed only a trend to higher levels in ACCs (both $P=0.13$; Fig. 1). The other evaluated factors (NOTCH1, HES5, CTNNB1, and LEF1) were similar in the three groups (NAGs, ACAs, and ACCs).

Concerning the relationship with clinical parameters, we observed a positive correlation between tumor size and mRNA levels of CTNNB1 $(R=0.41, P<0.005)$, HES1 $(R=0.37, P<0.01)$, and HEY2 $(R=0.37, P<0.05)$. However, no other significant correlations were observed between the mRNA expression of the investigated markers and clinical or histopathological parameters (ENSAT tumor stage, Weiss score, Ki67, number of distant metastases, and hormone secretion status).

\section{Protein expression of JAG1, aNOTCH1, HEY2, and $\beta$-catenin}

Representative examples of JAG1, aNOTCH1, and HEY2 staining in adrenal tissue are shown in Fig. 2. Both aNOTCH1 and JAG1 staining were relatively inhomogeneous, with the percentage of positive cells ranging from 10 to $85 \%$ and from 15 to $90 \%$ respectively. In contrast, HEY2 staining had a homogeneous tissue distribution in the entire series ( $>50 \%$ positive cells in more than $90 \%$ of samples).

All three of the evaluated components of the Notch1 signaling pathway were more strongly expressed in ACCs than they were in the other subgroups. In particular, JAG1 protein was highly expressed (H-score $2-3$ ) in $10 \%$ of NAGs, $15 \%$ of ACAs, and $27 \%$ of ACCs $\left(P<0.0005\right.$ by $\chi^{2}$ test), and nuclear aNOTCH1 protein was highly expressed in $0 \%$ of NAGs, $8 \%$ of ACAs, and $13 \%$ of ACCs $(P<0.005)$. HEY2 protein, which exhibited stronger staining intensity, was highly expressed (H-score $2-3$ ) in 33\% of NAGs, $61 \%$ of ACAs, and $66 \%$ of ACCs $(P<0.005$; Table 1$)$. Figure 3 shows the comparison in terms of $\mathrm{H}$-score values among the three groups (NAGs, ACAs, and ACCs) for JAG1, aNOTCH1, and HEY2. On the other hand, nuclear $\beta$-catenin expression was detected in a larger proportion of NAGs and ACAs than ACCs $(P<0.0001)$.

Of note, JAG1 expression significantly correlated with both aNOTCH1 and HEY2 in NAGs and ACAs $(P<0.001$
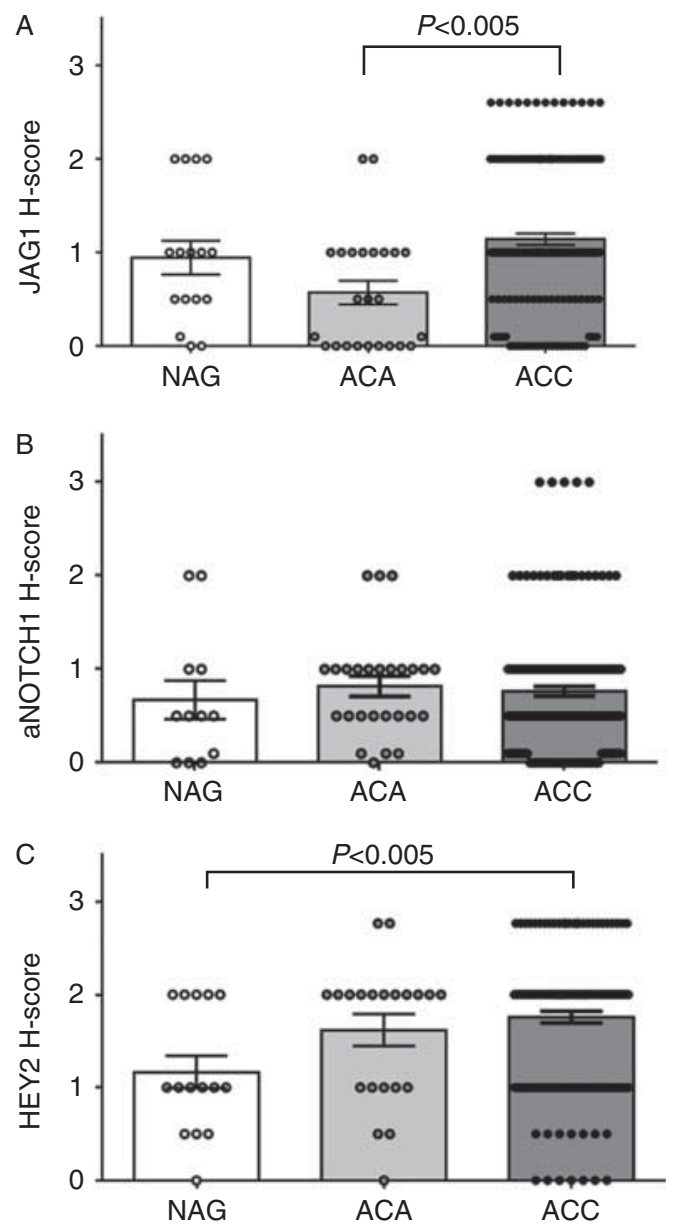

Figure 3

Evaluation of immunostaining as single and mean $\mathrm{H}$-score values ( \pm s.E.M.) in normal adrenal glands (NAGs, $n=16$ ), adrenocortical adenomas (ACAs, $n=27$ ), and carcinomas (ACCs, $n=178$ ). (A) Activated NOTCH1 (aNOTCH1), (B) JAG1, and (C) HEY2. Statistical analysis by one-way ANOVA.

and $P<0.05$ by $\chi^{2}$ test respectively) but not in ACCs (Supplementary Figure S2A, see section on supplementary data given at the end of this article), which suggests a conserved activation of the canonical Notch1 pathway in benign tumors but deregulated signaling in malignant tumors. Furthermore, nuclear $\beta$-catenin expression positively correlated with JAG1 only in ACAs $(P<0.005$ by $\chi^{2}$ test) and with HEY2 in both ACAs and ACCs (both $P<0.005$; Supplementary Figure S2B).

In the ACC group, no significant difference was observed among tumors that derived from first surgery $(n=140)$, from local recurrence $(n=26)$, or from metastasis $(n=14)$ for JAG1, aNOTCH1, and HEY2 protein expression. Among primary ACCs, JAG1 expression negatively correlated with tumor size $(R=0.18, P=0.056)$ and showed a trend toward higher expression in patients with early ENSAT tumor stages $(1-3, P=0.08)$ and in those

Published by Bioscientifica Ltd. 
with a lower number of distant metastases $(P=0.08)$. In contrast, HEY2 levels were higher in patients with metastatic tumors $(P<0.01)$.

JAG1 expression was higher in the nine ACCs that were affected by copy number gains in the previous SNP array analysis $(22 \%$ of cases with $\mathrm{H}$-score $<1 ; 44 \%$ with $\mathrm{H}$-score 1 ; and $33 \%$ with $\mathrm{H}$-score $2-3$ ) as compared to the seven ACCs with normal copy numbers ( $43 \%$ of cases with $\mathrm{H}$-score $<1 ; 57 \%$ with $\mathrm{H}$-score $1, P<0.005$; Supplementary Figure $\mathrm{S} 3$, see section on supplementary data given at the end of this article).

\section{Relationship between protein expression of JAG1, aNOTCH1, and HEY2 and clinical outcome}

Among ACCs, patients with higher JAG1 protein expression levels (H-score $\geq 1$ ) had a significantly longer OS (median 131 vs 30 months, $P<0.005$, HR 0.45 , 95\% CI 0.32-0.77) and PFS (median 37 vs 9 months, $P<0.005$, HR $0.51,95 \%$ CI $0.35-0.78$; Table 2). The significant after adjustment for ENSAT tumor stage $(P<0.01)$ and nuclear $\beta$-catenin expression $(P<0.01)$. Interestingly, ACCs with negative nuclear $\beta$-catenin expression $(n=62)$ had an impressively longer OS in cases of concomitant high JAG1 $(n=37$, median 126 months) than that in cases of concomitant low JAG1 $(n=25$, median 21 months, $P<0.01$, HR $0.34,95 \%$ CI 0.09-0.64; Fig. 4A).

Furthermore, the positive influence of high JAG1 expression on clinical outcome was particularly evident in the subgroup of patients who did not receive any pharmacological treatment $(n=26, P<0.05$, HR 0.12 , 95\% CI 0.05-0.61) but not in the subgroup of patients treated with mitotane monotherapy $(n=34, P=0.15$, HR $0.42,95 \%$ CI $0.19-1.27)$ or cytotoxic drugs $(n=62$, $P=0.64$, HR 0.87, 95\% CI 0.45-1.6).

In contrast, high HEY2 protein expression (H-score 2-3) was associated with a negative prognostic role in terms of OS (median 35 vs 86 months, $P=0.10$, HR $1.47,95 \%$ CI $0.94-2.26$ ) and PFS (median 9 vs 31 months, $P=0.13$, HR 1.34, 95\% CI 0.91-2.02; Table 2). The combined analysis

Table 2 Relationship between activated NOTCH1 (aNOTCH1), JAG1, and HEY2 protein expression and clinical outcome of patients with adrenocortical carcinoma ${ }^{a}$ (univariate analysis)

\begin{tabular}{|c|c|c|c|c|}
\hline & $n$ & $\begin{array}{l}\text { Median survival } \\
\text { (months) }\end{array}$ & HR $(95 \% \mathrm{Cl})$ & $P$ \\
\hline \multicolumn{5}{|l|}{ Overall survival } \\
\hline aNOTCH1 & & & $0.80(0.53-1.21)$ & 0.30 \\
\hline Low $(H$-score $<1)$ & 82 & 35 & & \\
\hline High & 57 & 45 & & \\
\hline JAG1 & & & $0.45(0.32-0.77)$ & $<0.005$ \\
\hline Low (H-score 0-1) & 101 & 30 & & \\
\hline High (H-score 2-3) & 36 & 131 & & \\
\hline HEY2 & & & $1.47(0.94-2.26)$ & 0.10 \\
\hline Low & 44 & 86 & & \\
\hline High & 83 & 35 & & \\
\hline Nuclear $\beta$-catenin & & & $1.71(1.05-2.99)$ & $<0.05$ \\
\hline Low & 53 & 89 & & \\
\hline High & 42 & 38 & & \\
\hline \multicolumn{5}{|c|}{ Free progression survival } \\
\hline aNOTCH1 & & & $0.86(0.58-1.23)$ & 0.41 \\
\hline Low $($ H-score $<1)$ & 82 & 13 & $0.51(0.35-0.78)$ & $<0.005$ \\
\hline $\begin{array}{l}\text { High } \\
\text { JAG1 }\end{array}$ & 57 & 10 & & \\
\hline Low (H-score 0-1) & 101 & 9 & & \\
\hline High (H-score 2-3) & 36 & 37 & & \\
\hline HEY2 & & & $1.37(0.92-2.07)$ & 0.13 \\
\hline Low & 44 & 31 & & \\
\hline High & 83 & 9 & & \\
\hline Nuclear $\beta$-catenin & & & $1.8(1.17-3.12)$ & $<0.05$ \\
\hline Low & 53 & 29 & & \\
\hline High & 42 & 9 & & \\
\hline
\end{tabular}

HR, hazard ratio; $95 \% \mathrm{Cl}$.

${ }^{a}$ Only ACC samples with complete available clinical data (total $n=137$ ). 

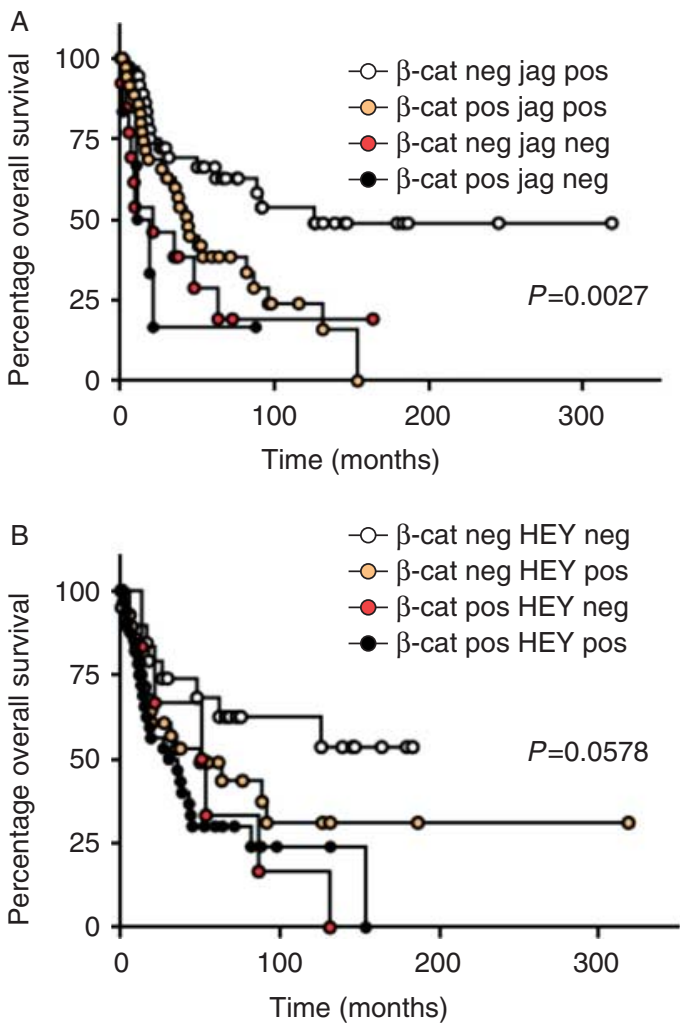

Figure 4

Overall survival analysis by Kaplan-Meyer curves in adrenocortical carcinomas: combination of (A) nuclear $\beta$-catenin and JAG1 protein expression $(n=95)$ and (B) nuclear $\beta$-catenin and HEY2 protein expression $(n=90)$.

with nuclear $\beta$-catenin expression showed a longer OS in the subgroup of patients with negative HEY 2 and negative nuclear $\beta$-catenin ( $n=21, P=0.058$; Fig. $4 \mathrm{~B})$.

Nuclear aNOTCH1 protein expression had no significant impact on OS or PFS (data not shown).

A multivariate regression analysis (by Cox regression test), including the expression of JAG1, aNOTCH1, HEY2, $\beta$-catenin, ENSAT tumor stage, and proliferation index Ki67, revealed an independent impact on OS only for JAG1 $(P=0.07$, HR 0.46$)$, tumor stage $(P<0.05$, HR 2.79$)$, and Ki67 $(P<0.005$, HR 3.81).

\section{Validation ACC cohort}

We also evaluated JAG1 and HEY2 protein expression in the independent cohort of 77 ACCs in order to validate the results of the first series. HEY2 was similarly expressed in the first series and in the validation series of ACCs, whereas JAG1 was more highly expressed in the validation series $(P<0.05$ vs first ACC series, $P<0.0005$ vs ACAs;
Supplementary Figure S4, see section on supplementary data given at the end of this article).

Moreover, despite the lower number of patients, the favorable impact of JAG1 protein levels on OS remained significant in the validation cohort of ACC patients (median 108 vs 50 months, $P<0.05$, HR $0.47,95 \%$ CI 0.24-0.94). Similarly to what we observed in the first series, high HEY2 expression levels were associated with a trend toward a worse prognosis (median OS 50 vs 100 months, $P=0.13$, HR $1.68,95 \%$ CI $0.86-3.37$ ). A graphical representation of the survival analysis for JAG1 in the first series and in the validation ACC series is shown in the Fig. 5.

\section{Discussion}

In a previous investigation that employed SNP array analysis, Notch signaling emerged as the most frequently altered pathway in adrenocortical neoplasias (Ronchi et al. 2013). In the present study, we therefore performed a more detailed analysis of components of this complex pathway in adrenal tumors at different levels. We found overexpression of the NOTCH1 ligand JAG1 and the downstream target of NOTCH1 HEY2 in ACCs as compared to NAGs or to benign adrenocortical lesions, at both the mRNA and the protein level. These results were also confirmed by immunohistochemistry in an independent validation cohort of ACCs. Furthermore, activated NOTCH1 protein expression was more frequently detected in malignant ACTs. However, no activating mutations in the PEST region of the NOTCH1 gene (Weng et al. 2004, Jundt et al. 2008, Ferrando 2010, Fabbri et al. 2011, Paganin \& Ferrando 2011, Rossi et al. 2012) were detected in ACCs. Of note, although gene expression of Notch1 pathway components correlated significantly in normal adrenals and adenomas, this relationship was lost in ACCs, which indicates dysregulation of Notch1 signaling in malignant tumors. Taken together, these observations confirm the activation of the Notch 1 pathway in adrenocortical neoplasias, particularly in ACCs.

Overexpression of the Notch ligand JAG1 was related to a copy number gain of the JAG1 gene in the subset of tumors for which SNP array data were available, which suggests that this mechanism may at least in part underlie the increased expression of JAG1. However, although high expression of JAG1 was more often found in ACCs than in ACAs or NAGs, it was characteristic of a subgroup of ACCs with a more differentiated phenotype and better prognosis. In particular, high JAG1 expression tended to be more frequent in smaller tumors, in early tumor stages,

Published by Bioscientifica Ltd 

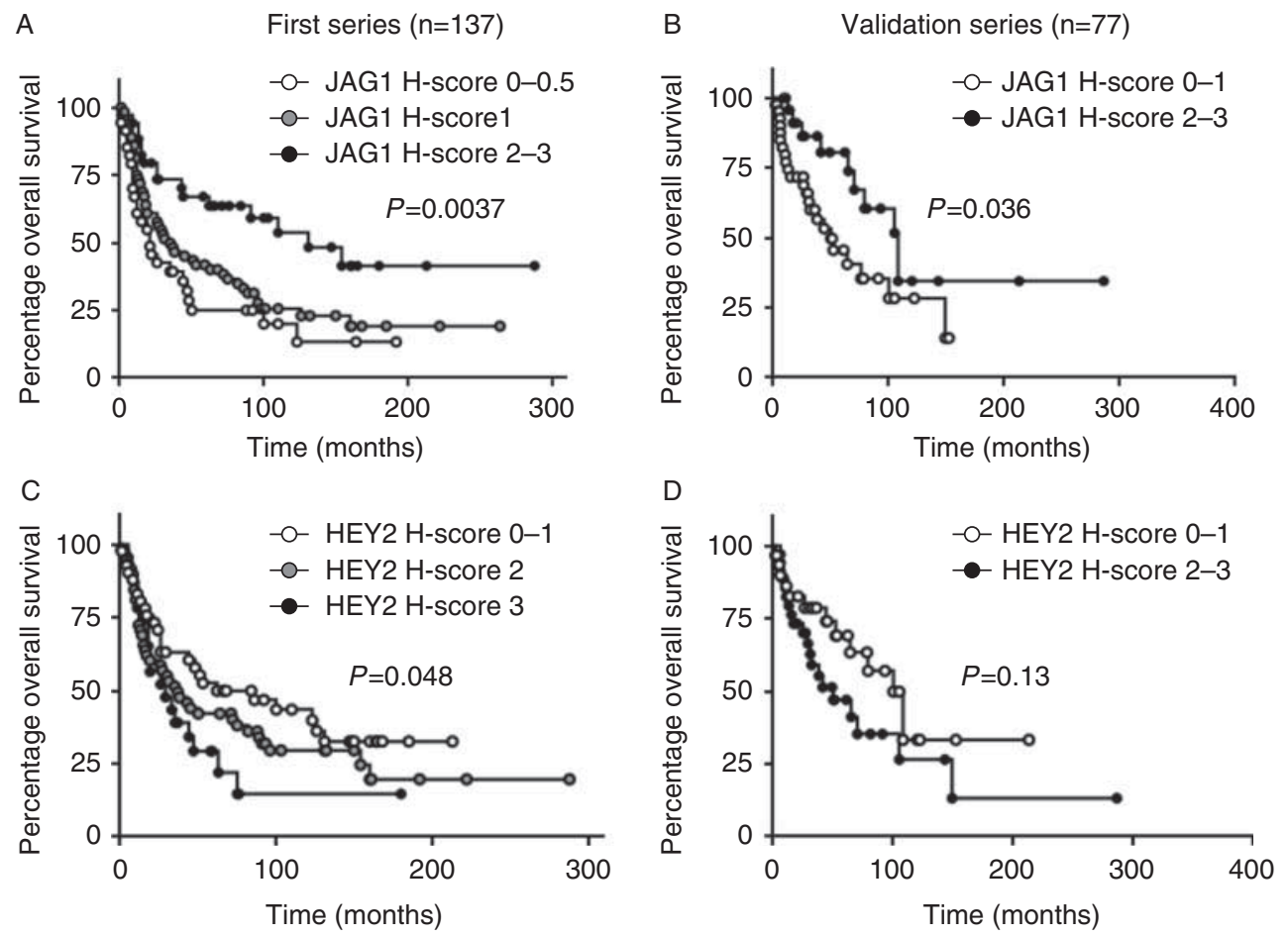

\section{Figure 5}

Overall survival analysis by Kaplan-Meyer curves in the first series of adrenocortical carcinomas (ACCs, A, C, $n=137$ ) and in the validation series

and in tumors with lower numbers of distant metastasis. Furthermore, it was associated with a better prognosis in terms of both improved PFS and improved OS. The favorable impact of JAG1 expression on general clinical outcome was also confirmed in the smaller validation ACC cohort. Therefore, high JAG1 indicates a less detrimental prognosis in ACCs.

Higher expression of JAG1 in ACCs as compared to ACAs and normal adrenals has already been described by Simon et al. (2012), but no prognostic impact was reported in that study. Instead, the authors showed that JAG1 overexpression in murine $\mathrm{Y} 1$ cells led to enhanced cell proliferation and aggressiveness. This discrepancy may be explained at least in part by the specific cellular context of Y1 cells and by species differences.

Intriguingly, the favorable impact of high JAG1 expression on clinical outcome was particularly evident in tumors with negative nuclear $\beta$-catenin expression, which supports the well-described importance of the interconnection of the $\mathrm{Wnt} / \beta$-catenin pathway (Balint et al. 2005, Crosnier et al. 2006, Wang et al. 2009a, Yamamizu et al. 2010, Kwon et al. 2011, Peignon et al. 2011, Kim et al. 2012, Ravindran \& Devaraj 2012, Gopalakrishnan et al. 2014).

( $\mathrm{B}$ and $\mathrm{D}, n=77$ ) for JAG1 protein expression ( $\mathrm{A}$ and $\mathrm{B}$ ) and HEY2 protein expression ( $C$ and $D$ ). $\beta$-cat, $\beta$-catenin.

Unlike JAG1, high HEY2 protein expression was instead a marker of a more aggressive tumor type with an inferior clinical outcome, although the impact on OS was not significant. This discrepancy with the JAG1 findings may reflect the profound dysregulation of the Notch pathway in ACCs and the role of additional influences downstream of Notch. The demonstration of Notch activation and the overexpression of its target genes in patients with advanced ACCs may become therapeutically relevant, considering the potential use of Notch-inhibiting drugs that act downstream of JAG1, such as $\gamma$-secretase inhibitors, which are currently under investigation alone and in combination in clinical trials for Notch-dependent solid tumors (Richter et al. 2014, Lee et al. 2015, LoConte et al. 2015, Messersmith et al. 2015), or more innovative compounds, such as receptor-blocking monoclonal antibodies (Supplementary Figure S1, see section on supplementary data given at the end of this article) (Hernandez Tejada et al. 2014).

Although the present investigation is the first attempt to analyze in more detail the Notch pathway in adrenocortical neoplasia, there remain clear limitations. The Notch pathway is highly complex, with four receptors, multiple ligands beyond JAG1, and several direct

Published by Bioscientifica Ltd. 
downstream targets in addition to the best-described Notch-HES/HEY family (Iso et al. 2003) that also participate in other important signaling pathways, such as the Wnt/ $\beta$-catenin pathway (e.g., CMYC, CCND1, and survivin) (Borggrefe \& Oswald 2009). Moreover, the Notch pathway interacts at different levels with other oncogenic pathways, such as the Wnt/ $\beta$-catenin signaling, the Shh, and the AKT/PI3K pathways (Supplementary Figure S1, see section on supplementary data given at the end of this article). Thus, a complete analysis would require the inclusion of many more components. However, the parameters evaluated in the present study are established key components of this pathway, and they allow important first insights into Notch signaling in adrenal neoplasias.

In summary, the activation of Notch1 signaling is demonstrated in ACCs, which suggests a potential role for Notch1 signaling in malignant adrenocortical transformation. However, JAG1 is overexpressed in a subgroup of ACCs that are characterized by a more differentiated phenotype and a better clinical outcome.

\section{Supplementary data}

This is linked to the online version of the paper at http://dx.doi.org/10.1530/ ERC-15-0163.

\section{Declaration of interest}

The authors declare that there is no conflict of interest that could be perceived as prejudicing the impartiality of the research reported.

\section{Funding}

This work was supported by a grant from the Wilhelm Sander Foundation (grant number 2012.095.1 to B Allolio). Furthermore, support was also received from the Deutsche Forschungsgemeinschaft DFG (grant numbers KR-43741/1-1 to M Kroiss and FA-466/4-1 to M Fassnacht) and from the IZKF Würzburg (grant number B-281 to M Fassnacht).

\section{Acknowledgements}

The authors are grateful to Martina Zink for excellent technical support and to Michaela Haaf for coordinating the German ACC Registry.

\section{References}

Assie G, Letouze E, Fassnacht M, Jouinot A, Luscap W, Barreau O, Omeiri H, Rodriguez S, Perlemoine K, Rene-Corail F et al. 2014 Integrated genomic characterization of adrenocortical carcinoma. Nature Genetics 46 607-612. (doi:10.1038/ng.2953)

Balint K, Xiao M, Pinnix CC, Soma A, Veres I, Juhasz I, Brown EJ, Capobianco AJ, Herlyn M \& Liu ZJ 2005 Activation of Notch1 signaling is required for $\beta$-catenin-mediated human primary melanoma progression. Journal of Clinical Investigation 115 3166-3176. (doi:10.1172/ JCI25001)

Borggrefe T \& Oswald F 2009 The Notch signaling pathway: transcriptional regulation at Notch target genes. Cellular and Molecular Life Sciences 66 1631-1646. (doi:10.1007/s00018-009-8668-7)

Capaccione KM \& Pine SR 2013 The Notch signaling pathway as a mediator of tumor survival. Carcinogenesis 34 1420-1430. (doi:10.1093/carcin/ bgt127)

Carvalho FL, Simons BW, Eberhart CG \& Berman DM 2014 Notch signaling in prostate cancer: a moving target. Prostate 74 933-945. (doi:10.1002/ pros.22811)

Chanrion M, Kuperstein I, Barriere C, El Marjou F, Cohen D, Vignjevic D, Stimmer L, Paul-Gilloteaux P, Bieche I, Tavares Sdos R et al. 2014 Concomitant Notch activation and p53 deletion trigger epithelialto-mesenchymal transition and metastasis in mouse gut. Nature Communications 5 5005. (doi:10.1038/ncomms6005)

Chillakuri CR, Sheppard D, Lea SM \& Handford PA 2012 Notch receptorligand binding and activation: insights from molecular studies. Seminars in Cell \& Developmental Biology 23 421-428. (doi:10.1016/j. semcdb.2012.01.009)

Crosnier C, Stamataki D \& Lewis J 2006 Organizing cell renewal in the intestine: stem cells, signals and combinatorial control. Nature Reviews. Genetics 7 349-359. (doi:10.1038/nrg1840)

Dai Y, Wilson G, Huang B, Peng M, Teng G, Zhang D, Zhang R, Ebert MP, Chen J, Wong BC et al. 2014 Silencing of Jagged1 inhibits cell growth and invasion in colorectal cancer. Cell Death \& Disease 5 e1170. (doi:10.1038/cddis.2014.137)

Du X, Cheng Z, Wang YH, Guo ZH, Zhang SQ, Hu JK \& Zhou ZG 2014 Role of Notch signaling pathway in gastric cancer: a meta-analysis of the literature. World Journal of Gastroenterology 20 9191-9199. (doi:10.3748/wjg.v20.i27.9191)

El Wakil A \& Lalli E 2011 The Wnt/ß-catenin pathway in adrenocortical development and cancer. Molecular and Cellular Endocrinology 332 32-37. (doi:10.1016/j.mce.2010.11.014)

Else T, Kim AC, Sabolch A, Raymond VM, Kandathil A, Caoili EM, Jolly S, Miller BS, Giordano TJ \& Hammer GD 2014 Adrenocortical carcinoma. Endocrine Reviews 35 282-326. (doi:10.1210/er.2013-1029)

Espinoza I \& Miele L 2013 Notch inhibitors for cancer treatment. Pharmacology \& Therapeutics 139 95-110. (doi:10.1016/j.pharmthera 2013.02.003)

Fabbri G, Rasi S, Rossi D, Trifonov V, Khiabanian H, Ma J, Grunn A, Fangazio M, Capello D, Monti S et al. 2011 Analysis of the chronic lymphocytic leukemia coding genome: role of NOTCH1 mutational activation. Journal of Experimental Medicine 208 1389-1401. (doi:10.1084/jem.20110921)

Fassnacht M, Johanssen S, Quinkler M, Bucsky P, Willenberg HS, Beuschlein F, Terzolo M, Mueller HH, Hahner S, Allolio B et al. 2009 Limited prognostic value of the 2004 International Union Against Cancer staging classification for adrenocortical carcinoma: proposal for a Revised TNM Classification. Cancer 115 243-250. (doi:10.1002/cncr. 24030)

Fassnacht M, Kroiss M \& Allolio B 2013 Update in adrenocortical carcinoma. Journal of Clinical Endocrinology and Metabolism 98 4551-4564. (doi:10.1210/jc.2013-3020)

Ferrando A 2010 NOTCH mutations as prognostic markers in T-ALL. Leukemia 24 2003-2004. (doi:10.1038/leu.2010.237)

Gallo-Payet N \& Battista MC 2014 Steroidogenesis-adrenal cell signal transduction. Comprehensive Physiology 4 889-964. (doi:10.1002/cphy. c130050)

Gaujoux S, Grabar S, Fassnacht M, Ragazzon B, Launay P, Libe R, Chokri I, Audebourg A, Royer B, Sbiera S et al. $2011 \beta$-catenin activation is associated with specific clinical and pathologic characteristics and a poor outcome in adrenocortical carcinoma. Clinical Cancer Research 17 328-336. (doi:10.1158/1078-0432.CCR-10-2006) 
Gopalakrishnan N, Saravanakumar M, Madankumar P, Thiyagu M \& Devaraj H 2014 Colocalization of $\beta$-catenin with Notch intracellular domain in colon cancer: a possible role of Notch1 signaling in activation of CyclinD1-mediated cell proliferation. Molecular and Cellular Biochemistry 396 281-293. (doi:10.1007/s11010-014-2163-7)

Gordon WR \& Aster JC 2014 Application and evaluation of anti-Notch antibodies to modulate Notch signaling. Methods in Molecular Biology 1187 323-333. (doi:10.1007/978-1-4939-1139-4_24)

Groth C \& Fortini ME 2012 Therapeutic approaches to modulating Notch signaling: current challenges and future prospects. Seminars in Cell \& Developmental Biology 23 465-472. (doi:10.1016/j.semcdb.2012.01.016)

Hernandez Tejada FN, Galvez Silva JR \& Zweidler-McKay PA 2014 The challenge of targeting notch in hematologic malignancies. Frontiers in Pediatrics 2 54. (doi:10.3389/fped.2014.00054)

Iso T, Kedes L \& Hamamori Y 2003 HES and HERP families: multiple effectors of the Notch signaling pathway. Journal of Cellular Physiology 194 237-255. (doi:10.1002/jcp.10208)

Jundt F, Schwarzer R \& Dorken B 2008 Notch signaling in leukemias and lymphomas. Current Molecular Medicine 8 51-59. (doi:10.2174/ 156652408783565540)

Kim HA, Koo BK, Cho JH, Kim YY, Seong J, Chang HJ, Oh YM, Stange DE, Park JG, Hwang D et al. 2012 Notch1 counteracts WNT/ $\beta$-catenin signaling through chromatin modification in colorectal cancer. Journal of Clinical Investigation 122 3248-3259. (doi:10.1172/JCI61216)

Kwon C, Cheng P, King IN, Andersen P, Shenje L, Nigam V \& Srivastava D 2011 Notch post-translationally regulates $\beta$-catenin protein in stem and progenitor cells. Nature Cell Biology 13 1244-1251. (doi:10.1038/ ncb2313)

Lee SM, Moon J, Redman BG, Chidiac T, Flaherty LE, Zha Y, Othus M, Ribas A, Sondak VK, Gajewski TF et al. 2015 Phase 2 study of RO4929097, a $\gamma$-secretase inhibitor, in metastatic melanoma: SWOG 0933. Cancer 121 432-440. (doi:10.1002/cncr.29055)

Li D, Masiero M, Banham AH \& Harris AL 2014 The notch ligand JAGGED1 as a target for anti-tumor therapy. Frontiers in Oncology 4254. (doi:10.3389/fonc.2014.00254)

Lobry C, Oh P \& Aifantis I 2011 Oncogenic and tumor suppressor functions of Notch in cancer: it's NOTCH what you think. Journal of Experimental Medicine 208 1931-1935. (doi:10.1084/jem.20111855)

LoConte NK, Razak AR, Ivy P, Tevaarwerk A, Leverence R, Kolesar J, Siu L, Lubner SJ, Mulkerin DL, Schelman WR et al. 2015 A multicenter phase 1 study of $\gamma$-secretase inhibitor RO4929097 in combination with capecitabine in refractory solid tumors. Investigational New Drugs 33 169-176. (doi:10.1007/s10637-014-0166-6)

Ma Y, Ren Y, Han EQ, Li H, Chen D, Jacobs JJ, Gitelis S, O'Keefe RJ, Konttinen YT, Yin G et al. 2013 Inhibition of the Wnt- $\beta$-catenin and Notch signaling pathways sensitizes osteosarcoma cells to chemotherapy. Biochemical and Biophysical Research Communications 431 274-279. (doi:10.1016/j.bbrc.2012.12.118)

Mazur PK, Riener MO, Jochum W, Kristiansen G, Weber A, Schmid RM \& Siveke JT 2012 Expression and clinicopathological significance of notch signaling and cell-fate genes in biliary tract cancer. American Journal of Gastroenterology 107 126-135. (doi:10.1038/ajg.2011.305)

McAuliffe SM, Morgan SL, Wyant GA, Tran LT, Muto KW, Chen YS, Chin KT, Partridge JC, Poole BB, Cheng KH et al. 2012 Targeting Notch, a key pathway for ovarian cancer stem cells, sensitizes tumors to platinum therapy. PNAS 109 E2939-E2948. (doi:10.1073/pnas. 1206400109)

Meng RD, Shelton CC, Li YM, Qin LX, Notterman D, Paty PB \& Schwartz GK $2009 \gamma$-secretase inhibitors abrogate oxaliplatin-induced activation of the Notch-1 signaling pathway in colon cancer cells resulting in enhanced chemosensitivity. Cancer Research 69 573-582. (doi:10.1158/0008-5472.CAN-08-2088)

Messersmith WA, Shapiro GI, Cleary JM, Jimeno A, Dasari A, Huang B, Shaik MN, Cesari R, Zheng X, Reynolds JM et al. 2015 A Phase I, DoseFinding Study in patients with advanced solid malignancies of the oral $\gamma$-secretase inhibitor PF-03084014. Clinical Cancer Research 21 60-67. (doi:10.1158/1078-0432.CCR-14-0607)

Nieman LK, Biller BM, Findling JW, Newell-Price J, Savage MO, Stewart PM \& Montori VM 2008 The diagnosis of Cushing's syndrome: an Endocrine Society Clinical Practice Guideline. Journal of Clinical Endocrinology and Metabolism 93 1526-1540. (doi:10.1210/ jc.2008-0125)

Paganin M \& Ferrando A 2011 Molecular pathogenesis and targeted therapies for NOTCH1-induced T-cell acute lymphoblastic leukemia. Blood Reviews 25 83-90. (doi:10.1016/j.blre.2010.09.004)

Peignon G, Durand A, Cacheux W, Ayrault O, Terris B, Laurent-Puig P, Shroyer NF, Van Seuningen I, Honjo T, Perret C et al. 2011 Complex interplay between $\beta$-catenin signalling and Notch effectors in intestinal tumorigenesis. Gut 60 166-176. (doi:10.1136/gut.2009. 204719)

Pfaffl MW 2001 A new mathematical model for relative quantification in real-time RT-PCR. Nucleic Acids Research 29 e45. (doi:10.1093/nar/ 29.9.e45)

Previs RA, Coleman RL, Harris AL \& Sood AK 2014 Molecular pathways: translational and therapeutic implications of the Notch signaling pathway in cancer. Clinical Cancer Research 21 955-961. (doi:10.1158/ 1078-0432.CCR-14-0809)

Radtke F \& Raj K 2003 The role of Notch in tumorigenesis: oncogene or tumour suppressor? Nature Reviews. Cancer 3 756-767. (doi:10.1038/ nrc1186)

Ranganathan P, Weaver KL \& Capobianco AJ 2011 Notch signalling in solid tumours: a little bit of everything but not all the time. Nature Reviews. Cancer 11 338-351. (doi:10.1038/nrc3035)

Ravindran G \& Devaraj H 2012 Aberrant expression of $\beta$-catenin and its association with $\Delta \mathrm{Np} 63$, Notch-1, and clinicopathological factors in oral squamous cell carcinoma. Clinical Oral Investigations 16 1275-1288. (doi:10.1007/s00784-011-0605-0)

Richter S, Bedard PL, Chen EX, Clarke BA, Tran B, Hotte SJ, Stathis A, Hirte HW, Razak AR, Reedijk M et al. 2014 A phase I study of the oral $\gamma$ secretase inhibitor R04929097 in combination with gemcitabine in patients with advanced solid tumors (PHL-078/CTEP 8575). Investigational New Drugs 32 243-249. (doi:10.1007/s10637013-9965-4)

Rizzo P, Miele L \& Ferrari R 2013 The Notch pathway: a crossroad between the life and death of the endothelium. European Heart Journal 34 2504-2509. (doi:10.1093/eurheartj/ehs141)

Ronchi CL, Sbiera S, Kraus L, Wortmann S, Johanssen S, Adam P, Willenberg HS, Hahner S, Allolio B \& Fassnacht M 2009 Expression of excision repair cross complementing group 1 and prognosis in adrenocortical carcinoma patients treated with platinum-based chemotherapy. Endocrine-Related Cancer 16 907-918. (doi:10.1677/ ERC-08-0224)

Ronchi CL, Sbiera S, Leich E, Tissier F, Steinhauer S, Deutschbein T, Fassnacht M \& Allolio B 2012 Low SGK1 expression in human adrenocortical tumors is associated with ACTH-independent glucocorticoid secretion and poor prognosis. Journal of Clinical Endocrinology and Metabolism 97 E2251-E2260. (doi:10.1210/jc.20122669)

Ronchi CL, Sbiera S, Leich E, Henzel K, Rosenwald A, Allolio B \& Fassnacht M 2013 Single nucleotide polymorphism array profiling of adrenocortical tumors - evidence for an adenoma carcinoma sequence? PLOS ONE 8 e73959. (doi:10.1371/journal.pone.0073959)

Rossi D, Rasi S, Fabbri G, Spina V, Fangazio M, Forconi F, Marasca R, Laurenti L, Bruscaggin A, Cerri M et al. 2012 Mutations of NOTCH1 are an independent predictor of survival in chronic lymphocytic leukemia. Blood 119 521-529. (doi:10.1182/blood-2011-09-379966)

Simon DP, Giordano TJ \& Hammer GD 2012 Upregulated JAG1 enhances cell proliferation in adrenocortical carcinoma. Clinical Cancer Research 18 2452-2464. (doi:10.1158/1078-0432.CCR-11-2371)

Steg AD, Katre AA, Goodman B, Han HD, Nick AM, Stone RL, Coleman RL, Alvarez RD, Lopez-Berestein G, Sood AK et al. 2011 Targeting the notch

Published by Bioscientifica Ltd. 
ligand JAGGED1 in both tumor cells and stroma in ovarian cancer. Clinical Cancer Research 17 5674-5685. (doi:10.1158/1078-0432.CCR11-0432)

Tissier F, Cavard C, Groussin L, Perlemoine K, Fumey G, Hagnere AM, Rene-Corail F, Jullian E, Gicquel C, Bertagna X et al. 2005 Mutations of $\beta$-catenin in adrenocortical tumors: activation of the Wnt signaling pathway is a frequent event in both benign and malignant adrenocortical tumors. Cancer Research 65 7622-7627.

Wang Z, Zhang Y, Li Y, Banerjee S, Liao J \& Sarkar FH 2006 Downregulation of Notch-1 contributes to cell growth inhibition and apoptosis in pancreatic cancer cells. Molecular Cancer Therapeutics 5 483-493. (doi:10.1158/1535-7163.MCT-05-0299)

Wang L, Qin H, Chen B, Xin X, Li J \& Han H 2007 Overexpressed active Notch1 induces cell growth arrest of HeLa cervical carcinoma cells. International Journal of Gynecological Cancer 17 1283-1292. (doi:10.1111/j.1525-1438.2007.00927.x)

Wang Z, Li Y, Banerjee S \& Sarkar FH 2009a Emerging role of Notch in stem cells and cancer. Cancer Letters 279 8-12. (doi:10.1016/j.canlet.2008. 09.030)

Wang Z, Li Y, Kong D, Banerjee S, Ahmad A, Azmi AS, Ali S, Abbruzzese JL, Gallick GE \& Sarkar FH 2009b Acquisition of epithelial-mesenchymal transition phenotype of gemcitabine-resistant pancreatic cancer cells is linked with activation of the notch signaling pathway. Cancer Research 69 2400-2407. (doi:10.1158/0008-5472.CAN-08-4312)
Wang Z, Li Y, Ahmad A, Azmi AS, Banerjee S, Kong D \& Sarkar FH 2010 Targeting Notch signaling pathway to overcome drug resistance for cancer therapy. Biochimica et Biophysica Acta 1806 258-267.

Weng AP, Ferrando AA, Lee W, Morris JP IV, Silverman LB, Sanchez-Irizarry C, Blacklow SC, Look AT \& Aster JC 2004 Activating mutations of NOTCH1 in human T cell acute lymphoblastic leukemia. Science 306 269-271. (doi:10.1126/science.1102160)

Westhoff B, Colaluca IN, D'Ario G, Donzelli M, Tosoni D, Volorio S, Pelosi G, Spaggiari L, Mazzarol G, Viale G et al. 2009 Alterations of the Notch pathway in lung cancer. PNAS 106 22293-22298. (doi:10.1073/ pnas.0907781106)

Yamamizu K, Matsunaga T, Uosaki H, Fukushima H, Katayama S, Hiraoka-Kanie M, Mitani K \& Yamashita JK 2010 Convergence of Notch and $\beta$-catenin signaling induces arterial fate in vascular progenitors. Journal of Cell Biology 189 325-338. (doi:10.1083/jcb. 200904114)

Yao J \& Qian C 2010 Inhibition of Notch3 enhances sensitivity to gemcitabine in pancreatic cancer through an inactivation of PI3K/Akt-dependent pathway. Medical Oncology 27 1017-1022. (doi:10.1007/s12032-009-9326-5)

Yoon SO, Zapata MC, Singh A, Jo WS, Spencer N \& Choi YS $2014 \gamma$ secretase inhibitors enhance vincristine-induced apoptosis in T-ALL in a NOTCH-independent manner. Apoptosis 19 1616-1626. (doi:10.1007/ s10495-014-1029-5)

Received in final form 11 May 2015

Accepted 15 May 2015

Made available online as an Accepted Preprint

15 May 2015
(C) 2015 Society for Endocrinology Printed in Great Britain 